\title{
The nature of singularity in multidimensional anisotropic Gauss-Bonnet cosmology with a perfect fluid
}

\author{
I.V. Kirnos \\ Tomsk State University, Tomsk, 634050 Russia \\ A.N. Makarenko \\ Tomsk State Pedagogical University, Tomsk, 634041 Russia \\ S.A. Pavluchenko \\ Special Astrophysical Observatory, Russian Academy of Sciences, Nizhnij Arkhyz, 369167 Russia \\ A.V. Toporensky \\ Sternberg Astronomical Institute, Moscow State University, Moscow, 119992 Russia

\begin{abstract}
We investigate dynamics of $(4+1)$ and $(5+1)$ dimensional flat anisotropic Universe filled
\end{abstract} \\ by a perfect fluid in the Gauss-Bonnet gravity. An analytical solutions valid for particular \\ values of the equation of state parameter $w=1 / 3$ have been found. For other values of \\ $w$ structure of cosmological singularity have been studied numerically. We found that for \\ $w>1 / 3$ the singularity is isotropic. Several important differences between $(4+1)$ and $(5+1)$ \\ dimensional cases are discussed.
}

\section{INTRODUCTION}

Recent several years show increasing interest to modified gravity both for $(3+1)$-dimensional Universe (which is mostly motivated by attempts to explain observed accelerated expansion of our Universe) and for multidimensional cosmological models which are more speculative, though they pose important questions on the nature of cosmological singularity and possible evolution of the Universe in its very early epoch. In the latter perspective the Lovelock gravity 1 is one of the very popular extension of General Relativity (GR) (see, for example, [2, 3, 4, 5, 6, 7, 8, 9, 10, 11, 12, 13, 14, 15]). It keeps the order of corresponding equations of motion unchanged with respect to GR, and, as it was recently claimed in [16], nice thermodynamical properties of GR survive in Lovelock gravity in contrast to, for example, $f(R)$ theories.

In the present paper we consider $(4+1)$ and $(5+1)$ dimensional anisotropic Universe. In these dimensions the only non-Einstein term in the Lovelock action is the famous Gauss-Bonnet 
combination. Power-law solutions which replace Kasner regime in the Gauss-Bonnet gravity in a flat anisotropic multidimensional Universe have been studied since the end of 80s [17]. However, all previous works have been devoted to vacuum solutions. Importance of the Kasner solution (which is a vacuum anisotropic solution in GR) follows from the fact that an ordinary barotropic fluid different from a massive scalar field is dynamically unimportant near a cosmological singularity. It means that we can still use the Kasner solution to describe initial singularity in a Universe filled by rather general kind of an ordinary matter. The goal of the present paper is to provide similar analysis for multidimensional Universe in the Lovelock gravity.

\section{MAIN EQUATIONS}

We consider a flat anisotropic metric in $(n+1)$-dimensional space-time. We are dealing with Einstein-Gauss-Bonnet gravity, and non-vacuum space-time. Lagrangian of this theory have a form

$$
\mathcal{L}=R+\alpha \mathcal{L}_{2}+\mathcal{L}_{M}
$$

where $R$ is Ricci scalar, $\mathcal{L}_{M}$ is the Lagrangian of matter fields and $\mathcal{L}_{2}$

$$
\mathcal{L}_{2}=R_{\mu \nu \alpha \beta} R^{\nu \mu \alpha \beta}-4 R_{\mu \nu} R^{\mu \nu}+R^{2}
$$

is the Gauss-Bonnet Lagrangian.

In the present paper we are interesting in the behavior mostly in the vicinity of the cosmological singularity, which allows us to take into account only corrections of the highest possible order. In our case it is the Gauss-Bonnet contribution, so we neglect Einstein terms. In the absence of matter sources this problem have been studied in [17, 18], in the present paper we take matter into account. The full Einstein - Gauss-Bonnet system shows a complicated behavior even in the vacuum case [19, 20], and we leave investigation of such system with matter for a future work.

We are working in the flat background, so the metric we considering has a form

$$
g_{\mu \nu}=\operatorname{diag}\left\{-1, a_{1}^{2}(t), a_{2}^{2}(t), \ldots, a_{n}^{2}(t)\right\}
$$

We use perfect fluid with the equation of state $p=w \rho$ as a matter source; after varying action obtained from (11) using the metric above and perfect fluid as a matter field, one can obtain the 
following equation of motion: there are $n$ dynamical equations and a constraint equation. The $j$-th dynamical equation has the form

$$
-3 \sum_{\substack{i, k, l, m \neq j \\ i<k<l<m}} H_{i} H_{k} H_{l} H_{m}-\sum_{i \neq j}\left(H_{i}^{2}+\dot{H}_{i}\right) \sum_{\substack{k, l \neq j, i \\ k<l}} H_{k} H_{l}=w \sigma \rho
$$

while constraint equation becomes

$$
3 \sum_{i<j<k<l} H_{i} H_{j} H_{k} H_{l}=\sigma \rho
$$

where $\sigma=2 \pi G / \alpha c^{4}$ is a combination of fundamental constants. We assume $\alpha>0$ in order to avoid instabilities in quantum theory. Also we need the continuity equation:

$$
\dot{\rho}+(\rho+p) \sum_{i=1}^{n} H_{i}=0
$$

To demonstrate the difference between $(4+1)$ and $(5+1)$ cases let us write down respective equation separately for these two cases. For $(4+1)$ case we have

$$
\left(\dot{H}_{b}+H_{b}^{2}\right) H_{c} H_{d}+\left(\dot{H}_{c}+H_{c}^{2}\right) H_{b} H_{d}+\left(\dot{H}_{d}+H_{d}^{2}\right) H_{b} H_{c}+w \sigma \rho=0
$$

as a dynamical equation (the rest three can be obtained by cyclic transmutation of indices) and the following constraint:

$$
3 H_{a} H_{b} H_{c} H_{d}=\sigma \rho
$$

For $(5+1)$ case we have

$$
\begin{aligned}
& 3 H_{b} H_{c} H_{d} H_{f}+\left(\dot{H}_{b}+H_{b}^{2}\right)\left(H_{c} H_{d}+H_{c} H_{f}+H_{d} H_{f}\right)+\left(\dot{H}_{c}+H_{c}^{2}\right)\left(H_{b} H_{d}+H_{b} H_{f}+H_{d} H_{f}\right) \\
& +\left(\dot{H}_{d}+H_{d}^{2}\right)\left(H_{b} H_{c}+H_{b} H_{f}+H_{c} H_{f}\right)+\left(\dot{H}_{f}+H_{f}^{2}\right)\left(H_{b} H_{c}+H_{b} H_{d}+H_{c} H_{d}\right)+w \sigma \rho=0
\end{aligned}
$$

as dynamical equations (rest four can be obtained the same way as for $(4+1)$ case) and 


$$
3\left[H_{a} H_{b} H_{c} H_{d}+H_{a} H_{b} H_{c} H_{f}+H_{a} H_{b} H_{d} H_{f}+H_{a} H_{c} H_{d} H_{f}+H_{b} H_{c} H_{d} H_{f}\right]=\sigma \rho
$$

as the constraint.

Now one can clearly see the differences between these two cases. First of all, the structure of the dynamical equations in the $(4+1)$ case (6) lacks the term with the product of four Hubble parameters. Indeed, this term in the $j$-th equation consists of the product of four Hubble parameters with indices different from $j$ and each other. In $(4+1)$ dimensions we have only three indices different from a given $j$, so this term is absent. In the $(5+1)$ case (8) there exits exactly four such indices, so one possible term exists. In higher dimensions with a bigger number of Hubble parameters the corresponding sum has more than one term. The second important difference exists in the structure of the constraint equations - in the $(4+1)$ case the left-hand side of (77) has only one term (all possible sums of four different Hubble parameters needed) while in the $(5+1)$ case (9) it has five of them. Both these features result in interesting differences in dynamical behavior of (4+1)and (5+1)-dimensional models (see below).

\section{POWER-LAW SOLUTIONS}

Now let us find some exact solutions with one particular anzatz. These solutions are of powerlaw-type, so scale factors take a form $a_{i}(t)=t^{p_{i}}$, after substituting Hubble functions $H_{i}=p_{i} / t$ and their derivatives $\dot{H}_{i}=-p_{i} / t^{2}$ into (3) and (4) one can rewrite constraint equation

$$
3 \sum_{i<j<k<l} p_{i} p_{j} p_{k} p_{l}=\sigma \rho_{0} t^{4-(1+w) \sum_{i} p_{i}}
$$

as well as equations of motion

$$
-3 \sum_{\substack{i, k, l, m \neq j \\ i<k<l<m}} p_{i} p_{k} p_{l} p_{m}-\sum_{i \neq j} p_{i}\left(p_{i}-1\right) \sum_{\substack{k, l \neq j, i \\ k<l}} p_{k} p_{l}=w \sigma \rho_{0} t^{4-(1+w) \sum_{i} p_{i}}
$$

there $\rho_{0}$ is the matter density at some given moment of time.

It is clear that if $\rho \neq 0$ then these equations can be solved only under condition

$$
\sum_{i} p_{i}=\frac{4}{1+w}
$$


Hence, field equations are

$$
\begin{gathered}
3 \sum_{i<j<k<l} p_{i} p_{j} p_{k} p_{l}=\sigma \rho_{0}, \\
-3 \sum_{\substack{i, k, l, m \neq j \\
i<k<l<m}} p_{i} p_{k} p_{l} p_{m}-\sum_{i \neq j} p_{i}\left(p_{i}-1\right) \sum_{\substack{k, l \neq j, i \\
k<l}} p_{k} p_{l}=w \sigma \rho_{0} .
\end{gathered}
$$

In Einstein gravity Kasner solution (for vacuum) implies $\sum_{i} p_{i}=1$, and such a condition conserves in Jacobs solution (for maximally stiff fluid) (for Kasner and Jacobs solutions see e. g. $[21,22]$ ). Assume now that similarly in Gauss-Bonnet gravity condition $\sum_{i} p_{i}=3$ (obtained earlier for vacuum) conserves for some kind of matter. One should keep in mind, though, that for vacuum $(4+1)$ case we still have $\sum_{i} p_{i}=3$ but under condition that one of $p_{i}$ is always zero - indeed, using anzatz above to Eq. (17) one can see that one of $p_{i}$ should be always zero.

From $\sum_{i} p_{i}=3$ and (12) one can obtain $w=1 / 3$. Translate now equations (13) and (14) while taking $w=1 / 3$ and $\sum_{i} p_{i}=3$ into account. For example,

$$
\begin{aligned}
24 & \sum_{i<j<k<l} p_{i} p_{j} p_{k} p_{l}=\sum_{i} p_{i} \sum_{j \neq i} p_{j} \sum_{k \neq i, j} p_{k} \sum_{l \neq i, j, k} p_{l}= \\
= & \sum_{i} p_{i} \sum_{j \neq i} p_{j} \sum_{k \neq i, j} p_{k}\left(3-p_{i}-p_{j}-p_{k}\right)= \\
= & 3 \sum_{i} p_{i} \sum_{j \neq i} p_{j} \sum_{k \neq i, j} p_{k}-3 \sum_{i} p_{i}^{2} \sum_{j \neq i} p_{j} \sum_{k \neq i, j} p_{k}=\ldots= \\
= & 81-54 \sum_{i} p_{i}^{2}+24 \sum_{i} p_{i}^{3}-6 \sum_{i} p_{i}^{4}+\left(\sum_{i} p_{i}^{2}\right)^{2} .
\end{aligned}
$$

At that all equations (13), (14) turns out to be identical with each other. Any of them takes a form

$$
81-54 \sum_{i} p_{i}^{2}+24 \sum_{i} p_{i}^{3}-6 \sum_{i} p_{i}^{4}+\left(\sum_{i} p_{i}^{2}\right)^{2}=8 \sigma \rho_{0} .
$$

Therefore, with $w=1 / 3$ considered metric with pointed above anzatz will be an exact solution only if

$$
\sum_{i} p_{i}=\frac{4}{1+w}=3, \quad 3 \sum_{i<j<k<l} p_{i} p_{j} p_{k} p_{l}=\sigma \rho_{0} .
$$

Cases $n=4$ and $n=5$ were investigated for arbitrary $w$. At $n=4$ there are no other anisotropic power-law solutions with nonzero energy density, but for $n=5$ there is the following solution:

$$
p_{1}=p_{2}=p_{3} \equiv p, \quad p_{4}=p_{5}=-\frac{1}{2} p
$$




$$
w=-\frac{p-2}{p}, \quad \sigma \rho_{0}=-\frac{3}{4} p^{4} .
$$

This solution have a remarkable feature: if 3 visible dimensions expanse isotropically then extra dimensions contract isotropically. However, here $\sigma \rho_{0}<0$, therefore, since $\sigma=\frac{2 \pi G}{\alpha c^{4}}$ either $\rho_{0}<0$, or $\alpha<0$. The latter means no global energy minimum and hence instability in quantum theory. So this solution seems to have no physical meaning; we wrote it down here just for mathematical completeness.

\section{NUMERICAL ANALYSIS}

In order to understand what happens for $w \neq 1 / 3$ we remind a reader similar situation in the Einstein gravity. Kasner solution gives us a condition $\sum_{i} p_{i}=1$ which indicates that the volume expands linearly with time, and, correspondingly, the matter density in the vicinity of Kasner regime decreases as $\rho \sim V^{-w-1} \sim t^{-w-1}$. On the other hand, left-hand side of equations of motion decreases as $t^{-2}$. These two scaling rates coincide at $w=1$ (the Jacobs solution). For $w<1$ a small amount of matter becomes dynamically unimportant for evolution towards a cosmological singularity, and ultimately disturbs the Kasner solution for an expanding Universe.

Of course, in the Einstein gravity we know the situation much better, because the dynamical equations describing Bianchi-I Universe with a perfect fluid can be solved exactly. This solution tells us that the Kasner regime is the unique past attractor in the case $w<1$ independently of the initial conditions, while isotropic regime is the unique future attractor [22]. Though $w$ can not exceed the value of 1 for any stable "physical" matter, there are some situation (for example in brane cosmology [23]) where the dynamics can be described as it would be driven by a matter with an effective $w>1$, and in this case the picture is the opposite one with past isotropic and future Kasner attractors [24, 25]].

In the Gauss-Bonnet gravity the situation is as follows. Left-hand sides of Eqs. $(3,4)$ scales as

$t^{-4}$ in vacuum, while volume increases as $t^{3}$. Adding a matter we obtain $\rho \sim V^{-w-1} \sim t^{-3 w-3}$ if the density of matter is small enough to keep the volume expansion rate close to its vacuum value. Comparing these two rates we see that the power-law vacuum solution is destroyed by matter if $w>1 / 3$ for a contracting Universe and for $w<1 / 3$ during expansion. On the other hand, the power-law solution is stable with respect to adding a small amount of matter with $w<1 / 3$ for contracting and with $w>1 / 3$ for expanding Universe.

In order to study global stability of the power-law solution and check whether its instability 

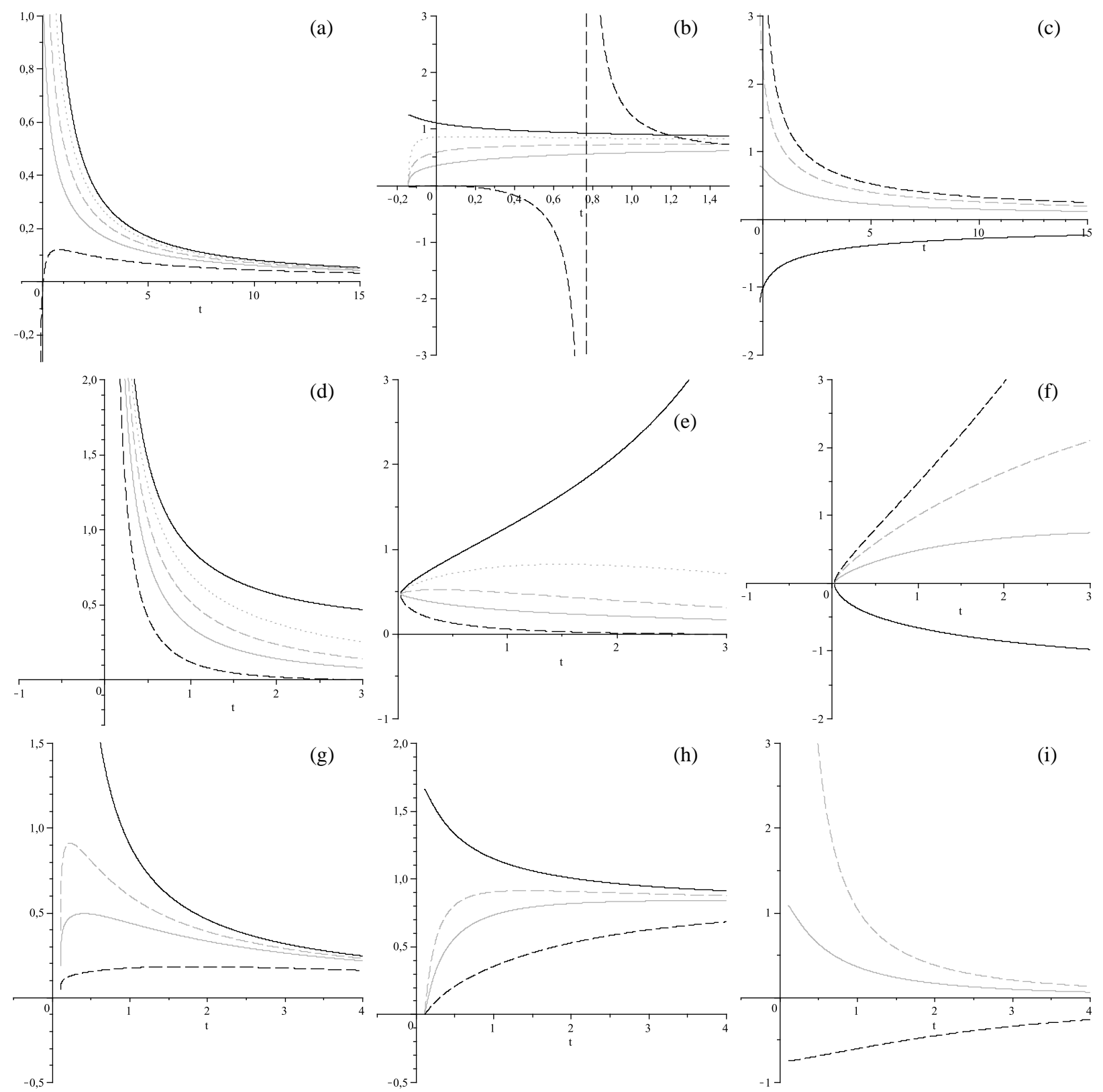

FIG. 1: Behavior of individual Hubble parameters (a, d, g), Kasner exponents (b, e, h) and relative Hubble differences (c, f, i) for [(5+1), w<1/3] (first row), [(5+1), w>1/3] (middle row) and [(4+1), w<1/3] (last row) cases. Note that $x$-axis $t$ is not a cosmological time, but rather an internal parameter of our numerical calculations. This implies for both Fig. 1 and Fig. 2.

for appropriate values of $w$ leads to isotropisation we provide numerical analysis of the system (3, 4) for various initial conditions and $w$. The results we have found are different for $(4+1)$ - and $(5+1)$-dimensions. 

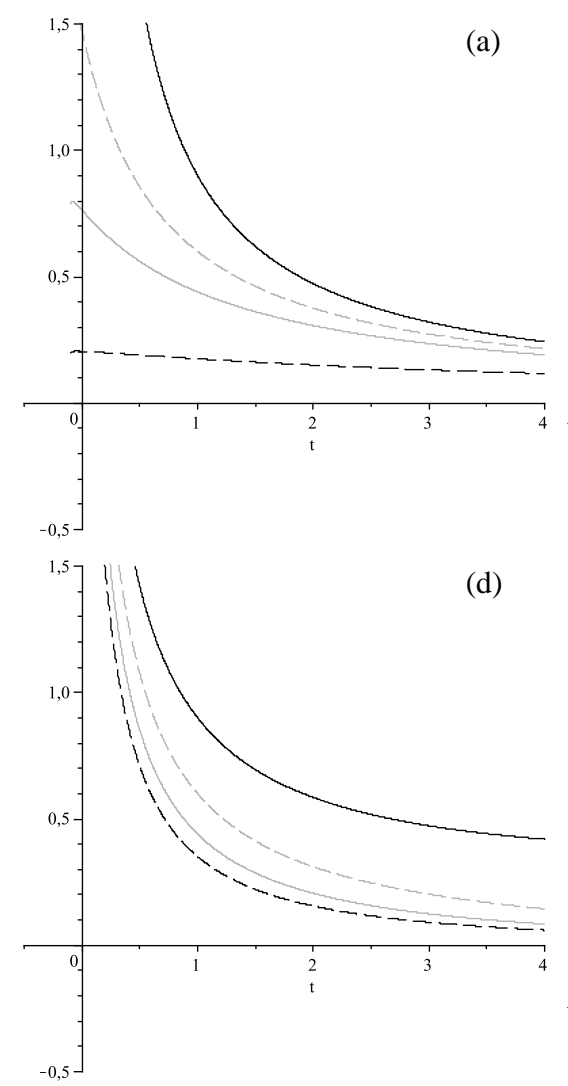
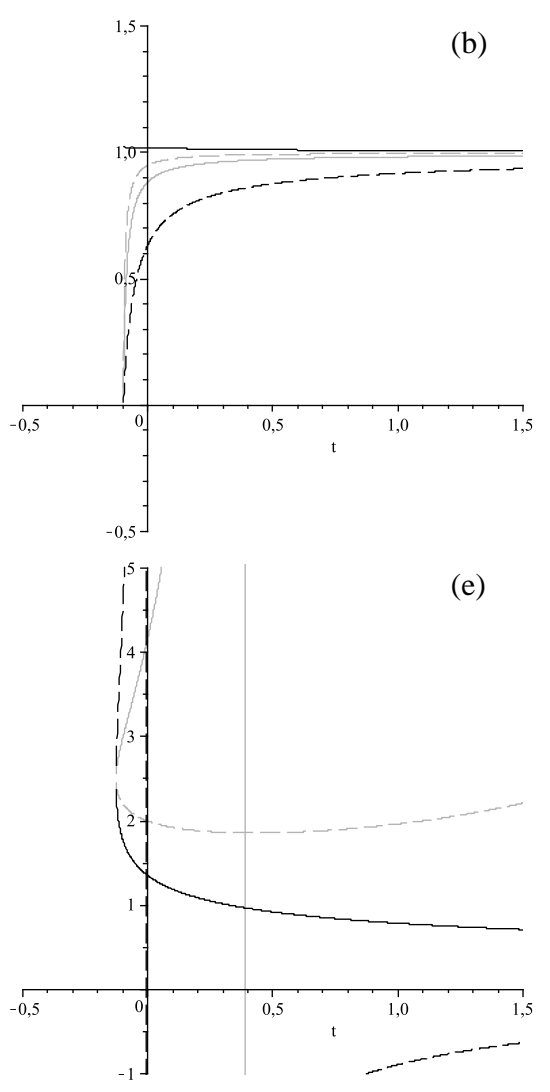

(c)
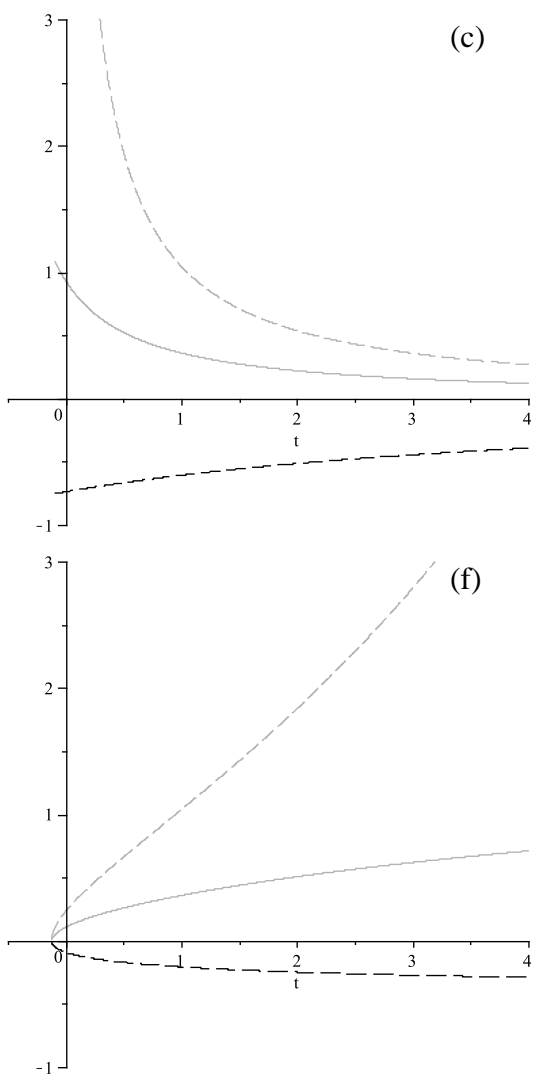

FIG. 2: Behavior of individual Hubble parameters (a, d), Kasner exponents (b, e) and relative Hubble differences (c, f) for $[(4+1), w=-0.01]$ (first row), [(4+1), $w>1 / 3]$ (last row) cases.

In the $(5+1)$-dimensional case the situation is similar to the General Relativity dynamics described above. In Fig. 1 (a, b, c) we plot typical behavior of power indices for $w<1 / 3$. There we have individual Hubble parameters in (a) panel, individual Kasner exponents in (b) and relative differences $\left(H_{i}-H_{1}\right.$ where $\left.i \neq 1\right)$ in (c) panel. We have found that for all studied initial conditions the past singularity is anisotropic and described by above mentioned power-law solution. And although in Fig. 1(b) four Kasner exponents tends to the same value (which is equal to zero), from Fig. 1 (c) one can verify that the singularity is anisotropic - differences are non-zero and not equal to each other. On the other hand, the singularity is isotropic (again, independently of initial conditions) if $w>1 / 3$ (see Fig. $1(\mathrm{~d}, \mathrm{e}, \mathrm{f})$ ). In this case one can see that all Kasner exponents tend to the same and non-zero value (Fig. 1 (e)) and Hubble differences all tend to zero (Fig. 1 (f)).

The situation in $(4+1)$ dimensions is more interesting. First of all, Eq. (6) indicates that while in the vacuum case at least one Hubble parameter should vanish, even a small amount of matter makes solutions with vanishing Hubble parameters impossible. This means that an arbitrary small 
$\rho$ changes properties of the solution significantly and can not be treated as a perturbation at any given moment of time. Thus, dynamics of $(4+1)$ dimensional Bianchi-I Universe can show features completely different from those in the vacuum case.

We have found such qualitative difference in case of pressureless matter $w=0$. It is easy to see that the conditions $p_{1}=p_{2}=p_{3}=p_{4}=1$ satisfy the system $(5,6)$ (we use $p_{i}=-H_{i}^{2} / \dot{H}_{i}$ ), providing an exact solution, which describes anisotropic Universe with constant (given by initial data and not changing with time) ratio of Hubble parameters. Note that for a higher-dimensional Universe a product of four Hubble parameters in the Eq.(5) destroys this solution, so it is valid only in $(4+1)$ dimensions.

Typical behavior of power indices for other $w<1 / 3$ is shown in Fig. 1 (g, h, i). It is quite similar to the $(5+1)$ case with $w<1 / 3$, but there are some differences, like tending some Hubble parameters to zero. In theories with Einstein term in the action this feature can violate dominance of Gauss-Bonnet contribution near a singularity, so this case needs more work. Initial singularity appears to be anisotropic with at least one of the Hubble parameters tending to zero. The last property is absent for $w=0$, and this value is an exceptional one: any nonzero $w$ leads to a qualitatively similar pictures, as it can be seen in Fig. 2 (a, b, c) plotted for $w=-0.01$. Since $w=-0.01$ satisfy $w<1 / 3$ condition, it looks the same with "usual" $[(4+1), w<1 / 3]$ case. With $w \rightarrow 0$ the sudden drop of Kasner exponents to zero becomes sharper and sharper, while in limit $w=0$ it disappears, and all Kasner exponents become equal to unity all the time, as explained above.

On the other hand, we have found that independently of initial conditions and the equation of state parameter, the initial singularity for the case of $w>1 / 3$ is isotropic (see Fig. $2(\mathrm{~d}, \mathrm{e}, \mathrm{f})$ ). The situation is the same as for $[(5+1), w>1 / 3]$ case.

\section{CONCLUSIONS}

We have studied the cosmological dynamics of a flat anisotropic multidimensional universe filled with a barotropic fluid in the Gauss-Bonnet gravity. Power-law analytical solutions existing for particular value of the equation of state parameter $(w=1 / 3)$ which are analogous to the Jacobs solution in the General Relativity existing for $w=1$ have been found.

In the $(5+1)$-dimensional case this analogy exists also for other values of $w$, leading to isotropic nature of the initial singularity for $w>1 / 3$ and anisotropic singularity for $w<1 / 3$. In $(4+1)$ 
dimensions the picture is less clear. First of all, an exceptional solution with constant anisotropy exists for $w=1$. Second, though our numerical results show clearly that singularity for $w<1 / 3$ is anisotropic, some Hubble parameters tend to zero, indicating that neglected Einstein term in the action may be important in this case. This issue requires special investigation. In case of $w>1 / 3$ singularity is isotropic in $(4+1)$ as well as in $(5+1)$ dimensions.

\section{Acknowledgments}

This work is partially supported by RFBR grant 08-02-00923 and scientific school grants 2553.2008.2 and 4899.2008.2.

[1] D. Lovelock, J. Math. Phys. 12, 498 (1971).

[2] N. Deruelle and L. Farina-Busto, Phys. Rev. D41, 3696 (1990).

[3] T. Kitaura and J. Wheeler, Phys. Rev. D48, 667 (1993).

[4] S. Nojiri, S. Odintsov, and S. Ogushi, Int. J. Mod. Phys. A17, 4809 (2002).

[5] T. Torii and H. Maeda, Phys. Rev. D71:124002 (2005).

[6] T. Torii and H. Maeda, Phys. Rev. D72:064007 (2005).

[7] H. Maeda, Phys. Rev. D73:104004 (2006).

[8] M. Aiello, R. Ferraro, and G. Giribet, Class. Quant. Grav. 22, 2579 (2005).

[9] J. Grain, A. Barrau, and P. Kanti, Phys.Rev. D72: 104016 (2005).

[10] M. Nozawa and H. Maeda, Class. Quant. Grav. 23, 1779 (2006).

[11] R. Cai and N. Ohta, Phys. Rev. D74:064001 (2006).

[12] E. Elizalde et al., Phys. Lett. B644, 1 (2007).

[13] C. Garraffo and G. Giribet, Mod. Phys. Lett. A23, 180 (2008).

[14] M. Dehghani and N.F arhangkhah, Phys. Rev. D78:064015 (2008).

[15] M. Dehghani, N. Bostani, and S. Hendi, Phys. Rev. D78:064031 (2008).

[16] A. Paranjape, S. Sarkar and T. Padmanabhan, Phys.Rev. D74:104015 (2006).

[17] N. Deruelle, Nucl. Phys. B327, 253 (1989).

[18] A. Toporensky and P. Tretyakov, Grav. Cosmol. 13, 207 (2007).

[19] R. Chingangban, M. Sami, A. Toporensky, and P. Tretyakov, Phys. Lett. B661, 162 (2008).

[20] S.A. Pavluchenko and A.V. Toporensky, Mod. Phys. Lett. A 24, 513 (2009).

[21] K. Jacobs, Astrophys. J. 153, 661 (1968). 
[22] Dynamical Systems in Cosmology, Ed. by J. Wainwright and G.F.R. Ellis (Cambrige University Press, Cambrige, 1997).

[23] P. Binetruy, C. Deffayet, and D.Langlois, Nucl Phys. B565, 269 (2000).

[24] R. Maartens, V. Sahni, and T.D. Saini, Phys. Rev. D63 063509 (2001)

[25] A. Toporensky, Class. Quant. Grav. 18, 2311 (2001). 